\title{
Construction of strongly regular graphs using affine designs
}

\author{
W. D. Wallis
}

Suppose there exist a balanced incomplete block design with $\lambda=1$ and an affine resolvable balanced incomplete block design, the two designs having the same replication number. Combining these designs we construct two strongly regular graphs. This is applied to give a new family of design graphs $((\nu, k, \lambda)$-graphs). Finally, we show that for any prime power $n$ there are two non-isomorphic design graphs with $v=n^{2}(n+2), k=n(n+1)$ and $\lambda=n$.

We have discussed design graphs, under the name " $(v, k, \lambda)$-graphs", in (for example) [6]. In this paper we give a construction method for the more general strongly regular graphs; we then discuss the particular cases when the graphs constructed are design graphs. We get two known classes of graphs and infinitely many new graphs. In particular we obtain the graphs with parameters $v=n^{2}(n+2), k=n(n+1), \lambda=n, n$ a prime power, which were discussed by Ahrens and Szekeres in [1]. We prove the existence of a pair of non-isomorphic graphs with these parameters for every suitable $n$.

The first two sections contain definitions which are needed later. General references for these are [3] and [5]. Material on strongly regular graphs appears in [2].

\section{Basic definitions}

A balanced incomplete block design (BIB design) with parameters

Received 24 August 1970. 
$(v, b, r, k, \lambda)$ is a scheme for selecting $b$-sets (called blocks) from a $v$-set of objects (called treatments) such that any treatment belongs to $r$ blocks and any pair of treatments belongs to $\lambda$ blocks. A symmetric $\mathrm{BIB}$ design, or $(v, k, \lambda)$-configuration, is one for which $v=b$. BIB designs necessarily satisfy

$$
\lambda(v-1)=r(k-1), \quad b k=v_{r} .
$$

An Hadamard matrix of order $4 n$ is a square matrix of order $4 n$ with elements \pm 1 and with any two rows orthogonal. It exists if and only if a (4n-1,2n-1,n-1)-configuration exists.

Graphs in this paper are finite and undirected, without loops or multiple edges. Thus a graph $\underline{\underline{G}}=(V, X)$ is a set $V$ of vertices together with a set $X$ of edges, or unordered pairs of vertices. If $(x, y) \in X$ then we say $x$ is adjacent to $y$, written $x \sim y$. If $U$ is a subset of $V$, then the spanning subgraph of $U$ in $\underline{\underline{G}}(V, X)$ is $\mathrm{G}(U, Y)$, where $Y$ consists of all members of $X$ with both vertices in $U$. The complement of $\underline{\underline{G}}(V, X)$ is $\underline{\underline{G}}(V, \bar{X})$, where $\bar{X}$ consists of all possible edges which are not in $X$. The union of graphs is defined by

$$
\underline{\underline{G}}\left(V_{1}, X_{1}\right) \cup \underline{\underline{G}}\left(V_{2}, X_{2}\right)=\underline{G}\left(V_{1} \cup V_{2}, X_{1} \cup X_{2}\right) \text {. }
$$

Write $S_{x}=\{y: y \in V, y \sim x\}$. Then $\left|S_{x}\right|$ is the valency of $x$. $\underline{\underline{G}}$ is called regular if $\left|S_{x}\right|=k$ for every $x$ in $V$; if also constants. $\lambda_{1}$ and $\lambda_{2}$ exist such that

$$
\begin{aligned}
& \left|S_{x} \cap S_{y}\right|=\lambda_{1} \text { when } x \sim y, \\
& \left|S_{x} \cap S_{y}\right|=\lambda_{2} \text { otherwise, }
\end{aligned}
$$

then it is called a strongly regular graph with parameters $\left(v ; k ; \lambda_{1}, \lambda_{2}\right)$. In particular when $\lambda_{1}=\lambda_{2}=\lambda, \underline{G}$ is called a design graph or $(v, k, \lambda)$-graph; in this case the vertices of $\underline{G}$ as treatments and the sets $S_{x}$ as blocks form a $(v, k, \lambda)$-configuration. The complement of a strongly regular graph with parameters $\left(v ; k ; \lambda_{1}, \lambda_{2}\right)$ is strongly regular with parameters

$$
\left(v ; v-k-1 ; v-2 k+\lambda_{2}-2, v-2 k+\lambda_{1}\right) \text {. }
$$

The complete graph and null graph on a certain set of vertices are 
the graphs with, respectively, all vertices adjacent and no vertices adjacent. The $k$-partite graph $\underline{K}_{n_{1}}, n_{2}, \ldots, n_{k}$ is a graph on $n_{1}+n_{2}+\ldots+n_{k}$ vertices constructed as follows: the vertices are partitioned into subsets called components of sizes $n_{1}, n_{2}, \ldots$, and $n_{k}$; the induced subgraph of any component is null; vertices in different components are always adjacent. If $n_{1}=n_{2}=\ldots=n_{k}=n$, we denote the graph $\underline{\underline{K}}_{n}^{k}$. For example, $\underline{\underline{K}}_{3}^{2}$ is shown.

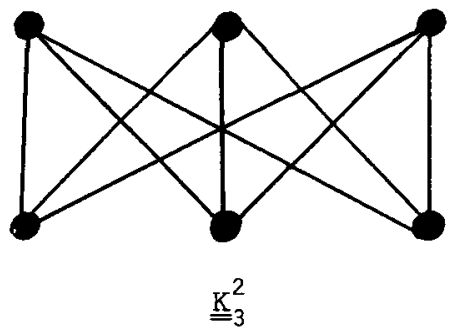

$\underline{K}_{n}^{k}$ is strongly regular with parameters

$$
(k n ;(k-1) n ;(k-2) n,(k-1) n)
$$

\section{Affine resolvable designs}

A BIB design with parameters $(v, b, r, k, \lambda)$ is called affine resolvable if its set of blocks can be partitioned into $r$ subsets or "parallel classes" of size $n$ such that each treatment occurs in exactly one block in each parallel class and such that two blocks from different parallel classes have $\mu$ common points for some fixed $\mu$. It is known $[3, p .73]$ that the parameters of an affine resolvable BIB design are (4) $v=n^{2} \mu, \quad b=n^{2} \mu+n(t+\mu), \quad r=n \mu+t+\mu, \quad k=n \mu, \lambda=t+\mu$, where the non-negative integer $t$ satisfies $\mu=(n-1) t+1$. Consequently we shall denote such a design as an $\operatorname{AR}(n, \mu)$. We shall refer to the blocks and treatments as lines and planes; lines in the same parallel class are called parallel, and clearly have no common points.

The designs $\operatorname{AR}(2, \mu)$ are precisely the family of designs discussed 
in [7]; consequently an $A R(2, \mu)$ exists if and only if there is an Hadamard matrix of order $4 \mu$ (that is, probably for all $\mu$ ).

An $\operatorname{AR}(n, 1)$ is precisely an affine plane of parameter $n$, so it is known to exist when $n$ is a prime power and known not to exist for certain values such as $n=6$. More generally, if we take the points and hyper-planes of affine $d$-space over $G F(q)$ as points and lines we obtain an $\operatorname{AR}\left(q, q^{d-2}\right)[3, p .72]$.

\section{Construction of strongly regular graphs}

THEOREM 1. Suppose there exists on $\operatorname{AR}(n, \mu)$ and there exists a $\mathrm{BIB}$ design $B$ with parameters $(v, b, r, k, 1)$, where

$$
r=n \mu+t+\mu, \mu=(n-1) t+1 .
$$

Then there is a strongly regular graph $G$ with parameters

$$
\left(v n^{2} \mu ;(v-1) n \mu ;(v-k) \mu+(k-2) n,(v-k) \mu\right) ;
$$

moreover the vertices of $G$ can be partitioned into $v$ subsets of size $n^{2} \mu$ whose induced subgraphs are nuzz.

Proof. First select $v$ disjoint sets $A^{l}, A^{2}, \ldots, A^{v}$ of size $n^{2} \mu$; the $v n^{2} \mu$ objects will be the vertices of $\underline{\underline{G}}$. Then construct designs $\underline{\underline{A}}^{l}, \underline{\underline{A}}^{2}, \ldots, \underline{\underline{A}}^{v}$, each of which is an $\operatorname{AR}(n, \mu)$ (these are not necessarily isomorphic), with $A^{\alpha}$ as the set of points of $\underline{\underline{A}}^{\alpha}$. Whenever $1 \leq \alpha \leq v$ and $1 \leq \pi \leq r$, the set of lines in the $\pi$-th parallel class of $\underline{\underline{A}}^{\alpha}$ is denoted $L_{\pi}^{\alpha}$.

With the $i$-th block $B_{i}=\{\beta, \gamma, \ldots, \delta\}$ of $\underline{\underline{B}}$ we associate a symbol $\left[\beta_{\rho}, \gamma_{\sigma}, \ldots, \delta_{\tau}\right]$, where $\rho, \sigma, \ldots, \tau$ are integers chosen in some way that each $\alpha_{\pi}$, where $l \leq \alpha \leq v$ and $l \leq \pi \leq r$, will occur in exactly one of the complete set of $b$ symbols. (This can be done since each $a$ occurs in exactly $r$ blocks of $\underline{\underline{B}} \cdot$ ) Corresponding to each block we construct a graph $\underline{G}_{i}$ which is the union of $n k$-partite graphs of type $\underline{K}_{n \mu}^{k}$ on disjoint sets of vertices: if $B_{i}$ has symbol 
$\left[\beta_{0}, \gamma_{\sigma}, \ldots, \delta_{\tau}\right]$ then the first components of the $n \underset{K_{n \mu}^{k}}{k}$ are the $n$ lines in $L_{\rho}^{\beta}$, the second components are the lines of $L_{\sigma}^{\gamma}, \ldots$, and the $k$-th components are the lines of $L_{\tau}^{\delta}$.

We define $\underline{\underline{G}}=U \underline{G} i$, and shall show that it has the desired properties.

Two vertices in $A^{\alpha}$ cannot lie in different components of the one $k$-partite graph, so they are not adjacent. Hence the various. $A^{\alpha}$ are $v$ disjoint subsets of size $n^{2} \mu$ whose induced subgraphs are null. If $x \in A^{\alpha}$ then the $r$ graphs in $G_{i}$ containing $x$ have no common points outside of $A^{\alpha}$ (as $\underline{\underline{B}}$ has last parameter 1 ); from (3) $x$ is adjacent to $(k-1) n \mu$ points of each $\underline{G}_{i}$ containing it; so $x$ has valency $r(k-1) n \mu=(\nu-1) n \mu$, for any $x$.

If $x$ and $y$ belong to $A^{\alpha}$ then they occur together in $\mu+t$ lines of $\stackrel{A}{\alpha}^{\alpha}$, so they occur in the same component in $\mu+t k$-partite graphs, and have $(k-1) n \mu$ common vertices adjacent to them both in each one; these vertices are all distinct. When $\alpha$ belongs to any other block $B_{j}$ of $\underline{B}, x$ and $y$ are in different $k$-partite graphs in $\underline{G}_{i}$; if $\beta$ is any other element of $B_{j}$ then the lines of $\underline{\underline{A}}^{\beta}$ which are adjacent to $x$ and $y$ are parallel, so they have no common points. So, in total, $x$ and $y$ are mutually adjacent to $(k-1) n \mu(\mu+t)$ vertices.

Say $x$ belongs to $A^{\beta}$ and $y$ to $A^{\gamma}, B \neq \gamma$. There will be one block $B_{i}$ of $B$ which contains both $B$ and $\gamma:$ either $x$ and $y$ are in the same $k$-partite graph in $\mathrm{g}_{i}$, whence they are adjacent and have $(k-2) n \mu$ mutually adjacent vertices in $\underline{G}_{i}$; or not, whence they are not adjacent and have no mutually adjacent vertices in $G_{i}$. This eliminates $k$ of the $A^{\alpha}$. Each of $x$ and $y$ are adjacent to one line ( $n \mu$ 
points) in any $\stackrel{\mathrm{A}}{\alpha}^{\alpha}$ where $\alpha \notin B_{i}$; these two lines of $\underline{\underline{A}}^{\alpha}$ are not parallel, since they correspond to different blocks, so they have $\mu$ common points by the properties of an $\operatorname{AR}(n, \mu)$. So $x$ and $y$ have $\mu(v-k)$ further mutually adjacent vertices.

Summarizing, two adjacent vertices have $\mu(v-k)+n(k-2)$ vertices mutually adjacent; two non-adjacent vertices have either $\mu(v-k)$ or $\mu(\mu+t) n(k-1)$. But applying (1) and (5) to $\underline{\underline{B}}$,

$$
\begin{aligned}
v-k & =r(k-1)-(k-1) \\
& =n(t+\mu)(k-1),
\end{aligned}
$$

so the graph is strongly regular.

THEOREM 2. Suppose there exist designs as in Theorem 1, which further satisfy

$$
k-2=\mu(n-2) \text {. }
$$

Then there is a strongly regular graph $\underline{\underline{H}}$ with parameters

$$
\left(v n^{2} \mu ;(v+n-1) n \mu-1 ; x+n^{2} \mu-2, x+2 n \mu\right)
$$

where $x=(v-2-\mu n+2 \mu) \mu$. Furthermore the vertices of $\underline{\underline{H}}$ can be partitioned into $v$ subsets of size $n^{2} \mu$ whose induced subgraphs are complete.

Proof. Construct $\underline{G}$ as in Theorem 1 , and in addition make all points in the same $A^{\alpha}$ adjacent. Call the new graph $\underline{\underline{\mathrm{H}}}$. If $S_{x}$ is the set of vertices adjacent to $x$ in $\underline{\underline{G}}$, then the set adjacent to $x$ in $\underline{\underline{H}}$ is

$$
S_{x} \cup\left(A^{\alpha} \backslash\{x\}\right)
$$

where $x \in A^{\alpha}$. As $S_{x}$ and $A^{\alpha}$ are disjoint, this set has order

$$
\left|S_{x}\right|+\left|A^{\alpha}\right|-1=(v+n-1) n \mu-1,
$$

so the graph is regular of the required valency. If $x \in A^{\alpha}$ and $y \in A^{\beta}$ the set of vertices adjacent to both $x$ and $y$ is 


$$
\begin{aligned}
& {\left[S_{x} \cup\left(A^{\alpha} \backslash\{x\}\right)\right] \cap\left[S_{y} \cup\left(A^{\beta} \backslash\{y\}\right)\right]} \\
& =\left[S_{x} \cap S_{y}\right] \cup\left[\left(A^{\alpha} \backslash\{x\}\right) \cap S_{y}\right] \cup\left[S_{x} \cap\left(A^{\beta} \backslash\{y\}\right)\right] \cup\left[\left(A^{\alpha} \backslash\{x\}\right) \cap\left(A^{\beta} \backslash\{y\}\right)\right] .
\end{aligned}
$$

The four sets enclosed by square brackets are disjoint. If $\alpha=\beta$, then the second and third sets have no elements and the fourth has $n^{2} \mu-2$. If $\alpha \neq \beta$ then the fourth is empty; the second and third have $n \mu$ elements each if $x$ and $y$ are non-adjacent and $n \mu-1$ each if they are adjacent, since $S_{x}$ includes one line of $A^{\beta}$ which contains $y$ if and only if $x \sim y$. The size of $S_{x} \cap S_{y}$ is given in Theorem 1 . With a little arithmetic we obtain the parameters shown in (8), provided (7) holds.

Naturally the theorems imply the existence of the complements of $\underline{G}$ and $\underline{\underline{H}}$ which are strongly regular; their parameters may be deduced from $(6),(8)$ and (2). In the complement of $\underline{\underline{G}}$ the $A^{i}$ induce complete graphs, while in the complement of $\underline{\underline{H}}$ they induce null graphs.

\section{Design graphs}

The graph $\underline{\underline{G}}$ of the preceding section is a design graph if and only if $k=2$. In that case $\underline{B}$ always exists.

COROLLARY 3. If there is an $\operatorname{AR}(n, \mu)$, then there is a design graph with parameters

$$
\left(n^{2} \mu(n \mu+t+\mu+1), n \mu(n \mu+t+\mu), n \mu(\mu+t)\right),
$$

where $\mu=(n-1) t+1$. In particular

(i) if there is an affine plane of parameter $n$, then there is a groph

$$
\left(n^{2}(n+2), n(n+1), n\right) \text {; }
$$

(ii) if there is an Hadamard matrix of order $4 \mu$, then there is a groph

$$
\left(16 \mu^{2}, 8 \mu^{2}-2 \mu, 4 \mu^{2}-2 \mu\right) ;
$$

(iii) if $n$ is a prime power greater than 2 and $d$ is an integer greater than 2 , then there is a graph 


$$
\left(\frac{n^{d}\left(n^{d}+n-2\right)}{n-1}, \frac{n^{d-1}\left(n^{d}-1\right)}{n-1}, \frac{n^{d-1}\left(n^{d-1}-1\right)}{n+1}\right) .
$$

The parameters (11) all seem to be new.

Part (ii) of the corollary appears in [4]. Note that a graph with parameters (10) implies the existence of a design graph

$$
\left(16 \mu^{2}-1,8 \mu^{2}, 4 \mu^{2}\right)
$$

by Theorem 4 of [6].

Graphs with parameters (9) with $n$ a prime power were constructed by Ahrens and Szekeres in [1]. Their graphs all have the following property: if $x$ and $y$ are any two adjacent vertices and $z$ and $t$ are mutually adjacent to $x$ and $y$, then $z$ is adjacent to $t$.

In our construction for (9) the parameters of $\underline{\underline{B}}$ include $v=n+2$ and $k=2$. There are $n$ lines in each parallel class of each $A^{\alpha}$. Suppose $x$ and $y$ are two given'adjacent vertices in our graph and $z \in A^{\beta}$ and $t \in A^{\gamma}$ are adjacent to both $x$ and $y$. Suppose the symbol corresponding to the block $\{\beta, \gamma\}$ of $\underline{\underline{B}}$ is $\left[\beta_{\sigma}, \gamma_{\tau}\right]$. Since $n \geq 2$, we have enough freedom to ensure that the lines containing $z$ and $t$ in $L_{\sigma}^{\beta}$ and $L_{\tau}^{\gamma}$ do not lie in the same 2-partite graph, ensuring that our graph is not isomorphic to the Ahrens-Szekeres solution.

COROLLARY. If $n$ is a prime power, there are at least two non-isomorphic grophs of parameters (9).

The other graphs constructed in Section 3 do not yield design graphs, except that the complement of the graph $\underline{\underline{H}}$ of Theorem 2 is a graph of parameters (10) when $n=k=2$.

\section{References}

[1] R.W. Ahrens and G. Szekeres, "On a combinatorial generalization of 27 lines associated with a cubic surface", J. Austral. Math. Soc. 10 (1969), 485-492. 
[2] R.C. Bose, "Strongly regular graphs, partial geometries and partially balanced designs", Pacific J. Math. 13 (1963), 389-419.

[3] P. Dembowski, Finite geometries (Ergebnisse der Mathematik und ihrer Grenzgebiete, Band 44, Springer-Verlag, Berlin, Heidelberg, New York, 1968).

[4] J.M. Geothals and J.J. Seidel, "Strongly regular graphs derived from combinatorial designs", Canad. J. Math. 22 (1970), 597-614.

[5] Frank Harary, Groph theory (Addison-Wesley, Reading, Massachusetts; London, Ontario, 1969).

[6] W.D. Wallis, "Certain graphs arising from Hadamard matrices", Buzz. Austral. Math. Soc. 1 (1969), 325-331.

[7] W.D. Wallis, "A note on quasi-symmetric designs", J. Combinatorial Theory 9 (1970), 100-101.

University of Newcastle,

Newcastle,

New South Wales. 